\title{
Intégration des contraintes ferroviaires dans les chantiers de mise au gabarit de tunnels
}

\section{J.-C. DAUMARIE \\ C. THUAUD}

SNCF

Direction de l'ingénierie

Département

des ouvrages d'art

6, avenue

François-Mitterrand

93574 La Plaine Saint-Denis

Jean-claude.daumarie@sncf.fr

Christine.thuaud@sncf.fr

L'évolution des transports et en particulier des transports de fret, conduit à prévoir, pour les tunnels ferroviaires, des gabarits de plus en plus grands. Les tunnels du patrimoine français, construits il y a plus de 100 ans, ne dégagent pas ces nouveaux gabarits et doivent donc être agrandis, au moyen de travaux de rescindement de leurs structures notamment. Ces travaux doivent se dérouler en perturbant le moins possible la circulation des trains. C'est pourquoi les études géotechniques doivent tenir compte non seulement des propriétés des sols ou roches environnant le tunnel, mais aussi de ces contraintes ferroviaires. Ces contraintes sont décrites dans cet article en présentant la modélisation de différentes phases des travaux avec des sollicitations correspondantes.

Mots-clés : tunnels ferroviaires, gabarits, rescindement, phasage, modélisation, calculs aux éléments finis.

\section{Integration of railways constraints during works for enlarging railways tunnels gauges}

Evolution of transportations, especially freight transportation, leads to forecast bigger and bigger gauges for railway tunnels. French tunnels built more than 100 years ago do not allow these new gauges and have to be enlarged with works such as localised reaming. These works have to be carried out without significantly affecting the normal traffic. That is why geotechnical investigations have to take into account not only the properties of soils or rocks that surround the tunnel, but also the different railway constraints. These constraints are described in this paper by presenting the modelling of different work phases with the corresponding loads.

Key words: railway tunnels, gauges, splitting, work phases, modeling, finite elements method. 


\section{Les tunnels du patrimoine français}

Le patrimoine des tunnels du réseau ferroviaire français comporte 1548 tunnels répartis sur $631 \mathrm{~km}$ de lignes, dont 1378 tunnels répartis sur $572 \mathrm{~km}$ de lignes exploitées. La période de grande activité de construction de ces ouvrages s'est étendue de 1850 à 1900, ce qui explique que l'âge moyen des tunnels soit de plus de 100 ans.

Pour chaque tunnel, la construction est un cas d'espèce qui dépend essentiellement des conditions géologiques (nature, caractéristiques et particularités des terrains, présence d'eau) et géotechniques, mais également des contraintes économiques au regard du coût et de la durée des travaux. D'autres paramètres ont pu intervenir, tels que la géométrie de l'ouvrage (longueur, pente, section) ou l'environnement avec les spécificités entraînées par les ouvrages en zone urbaine, en site classé, ou en zone de montagne, mais là encore, le rôle de la géologie et de la géotechnique a été et restera toujours prépondérant.

Les techniques de construction ont beaucoup évolué, en particulier depuis le milieu du XIX siècle, ce qui impose un effort important pour comprendre le comportement des structures, en particulier des terrains environnants, que n'éclaire plus toujours l'expérience actuelle des concepteurs et des constructeurs.

\section{2}

\section{La conception du dégagement des gabarits}

RFF et SNCF sont confrontés au besoin de faire circuler des convois de plus en plus grandes dimensions, pour transport de fret en particulier. De ce fait, les gabarits à dégager dans les tunnels sont de plus en plus importants, comme le montre la figure 1 comparant les gabarits d'origine et le gabarit le plus récent.

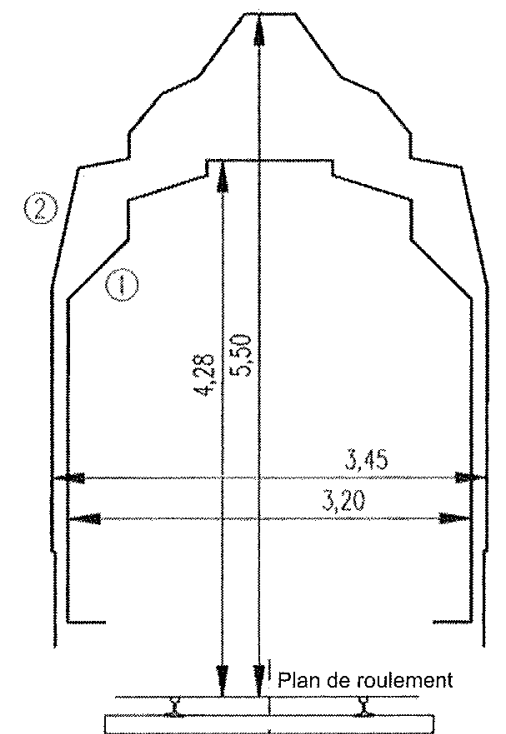

1. Gabarit des trains impériaux en 1863.

2. Gabarits GB1 UIC (B + français) et d'isolement

de pantographes et caténaire à courant $25 \mathrm{kV}$.
Différents itinéraires comportant des tunnels sont ainsi actuellement l'objet d'études de dégagement de gabarits. Ces études commencent naturellement par des études géométriques, constituées de relevés des profils des ouvrages et d'analyses du tracé des voies et des possibilités de le modifier. Ces études permettent d'évaluer la longueur et la profondeur des engagements de gabarit, et les dispositions à prendre en conséquence :

- interventions sur tracé et entraxe de voies ;

- abaissements localisés de la plate-forme ferroviaire, ou rescindements localisés de la voûte.

Ce n'est que lorsque les engagements de gabarits sont trop importants qu'interviennent alors des travaux de plus grande ampleur :

- abaissements importants de plate-forme ;

- rescindement de grande profondeur de la voûte.

Ces deux types de travaux impliquent dès lors une bonne connaissance des conditions géologiques et géotechnicues du massif environnant le tunnel. Mais l'organisation et la nature des travaux devront aussi s'adapter aux contraintes ferroviaires.

\section{Les contraintes ferroviaires}

Les travaux de mise au gabarit des tunnels ne peuvent se dérouler qu'en s'adaptant aux contraintes du trafic dans l'ouvrage, elles-mêmes dépendantes des conditions d'exploitation de l'itinéraire sur lequel se trouve le tunnel. Les investigations préalables aux travaux, caractéristiques de la conduite d'études géotechniques, comme on le détaille plus loin, sont, elles aussi, soumises à ces contraintes. On rappelle que les tunnels ferroviaires peuvent être à double voie ou à voie unique, et qu'une première des contraintes ferroviaires est de s'adapter à cette situation.

\section{S.}

\section{Tunnels à double voie}

Dans le cas d'un tunnel à double voie, plusieurs organisations de chantier sont possibles selon les conditions de circulation : interception totale d'une voie, voie unique temporaire, tronc commun temporaire avec une seule voie au centre, interception complète des deux voies.

\section{6.}

\section{Tunnels à voie unique}

Dans le cas d'un tunnel à voie unique, les organisations sont bien évidemment moins variées, l'alternative portant simplement sur le maintien ou non des circulations durant les travaux :

- maintien des circulations ferroviaires. L'organisation est alors assimilable à celle d'un chantier en tunnel à double voie se déroulant voie par voie; les travaux sont alors conçus pour se dérouler sur des intervalles de durées limitées, en dehors desquels les trains doivent pouvoir circuler en toute sécurité, mais éventuellement à vitesse réduite pendant la durée du chantier ; 
- coupure du trafic. L'organisation est alors assimilable à celle de travaux en tunnel à double voie également organisés sous coupure de ligne. Les contraintes strictement ferroviaires sont alors inexistantes, puisque aucune contrainte ne vient perturber les travaux, si ce n'est l'optimisation de la durée de façon à rétablir au plus vite le trafic.

Selon les possibilités de modifier l'exploitation normale, c'est-à-dire la fréquence et la répartition des trains, se dégageront donc diverses modalités de réalisation des travaux. En particulier, seront à prendre en compte les délais d'intervention pendant lesquels des travaux sont possibles mais aussi à l'issue desquels les trains devront pouvoir circuler en toute sécurité. Ceci signifie que les phasages des travaux de démolition puis de reconstruction devront permettre de respecter cette contrainte de mise en sécurité. Bien entendu, ces phasages devront prendre en compte les conditions géotechniques

\section{4}

\section{Les études géotechniques}

Les études géotechniques doivent prendre en compte :

- la nature des travaux imposés par l'objectif de dégagement de gabarit ;

- le phasage et le délai des travaux, imposés par les contraintes d'exploitation.

Plusieurs hypothèses de travaux sont à étudier, auxquelles doivent s'adapter non seulement le programme des investigations, mais aussi la conduite des calculs de dimensionnement. Les études doivent comporter la définition et l'interprétation des reconnaissances puis la modélisation du comportement des terrains.

\section{4.}

\section{Programme des investigations}

Les investigations consistent classiquement en investigations destructives, incluant sondages et essais in situ, et en investigations non destructives, incluant notamment toute la gamme des méthodes géophysiques.

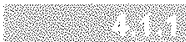 \\ Investigations destructives}

Les investigations destructives sont a priori seules à même de fournir de véritables données sur le comportement des terrains et des structures. Mais leur exécution occasionne de fortes perturbations au trafic ferroviaire, dont on rappelle qu'il doit le plus possible être préservé, y compris lors de cette phase d'étude.

La logistique associée aux travaux de sondages est en effet beaucoup plus lourde que dans le cas de constructions de tunnels ferroviaires ou routiers, ou même dans le cas d'investigations en tunnels routiers sous circulation. Les installations de sondages doivent être embarquées sur des trains se déplaçant sur les voies, pour pouvoir être intégrées dans le trafic ferroviaire (Fig. 2). L'implantation, puis le sondage lui-même se déroulent dans un espace confiné non seulement

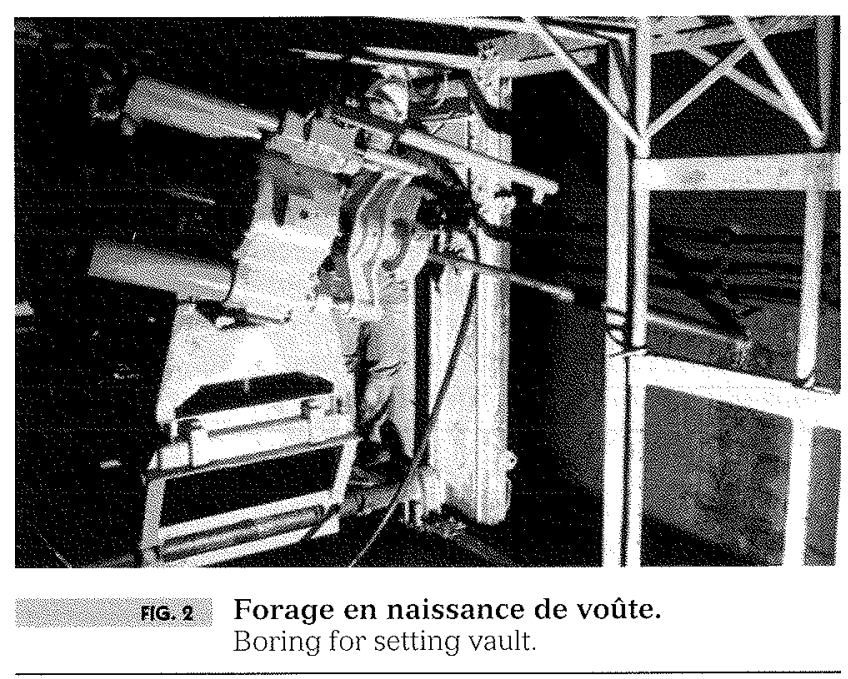

par l'installation sur voie, mais aussi par la géométrie de l'ouvrage, par l'éventuel maintien des circulations ferroviaires sur la voie contiguë et, donc, par la présence de courant d'alimentation électrique dans la caténaire toute proche.

\section{- Les sondages carottés}

Depuis 1962, la SNCF procède à la reconnaissance des tunnels par sondages carottés. En 2007, plus des deux tiers du patrimoine des tunnels de RFF sont déjà reconnus par carottage. Les sondeuses sont disposées sur un wagon, elles permettent une mise en station rapide et la reprise d'un carottage interrompu en fin d'interception des voies. Tous les points du profil transversal du tunnel doivent pouvoir être auscultés, y compris la base des piédroits afin d'apprécier le niveau d'encastrement des fondations par rapport au plan des voies.

L'exécution de carottages ascendants nécessite, pendant les phases de carottage et d'extraction, un matériel de forte capacité même si la longueur des carottages est limitée à quelques mètres. De ce fait, les sondages manuels sont réservés à des investigations de très faibles longueurs.

Pour la réalisation des sondages en plate-forme, un tubage provisoire est nécessaire à la traversée du ballast pour éviter les chutes de blocs lors de la remontée du train de tige en fin de première passe de carottage.

Les sondages carottés sont complétés et valorisés par le passage d'une sonde endoscopique ou vidéoendoscopique, permettant d'observer les parois du sondage et de repérer des discontinuités, vides, altération des matériaux, ainsi que la nature du contact entre la structure et l'encaissant. Bien que les sondages carottés donnent des observations ponctuelles, l'interpolation entre ces sondages permet d'établir les coupes géologiques transversales et longitudinales et fournit une première connaissance du massif encaissant permettant d'élaborer une interprétation géotechnique. Les sondages carottés sont le complément indispensable pour qualifier les observations et les anomalies déterminées par les techniques d'auscultation non destructive.

\section{- Les sondages destructifs}

Les sondages destructifs ne permettent pas d'obtenir de valeur des paramètres géotechniques du sol. 
Mais ils permettent à moindre coût (le rapport est de un pour trois avec le coût d'un carottage) de se renseigner sur l'homogénéité d'une formation ou sur la géométrie d'une discontinuité ou d'un contact entre formations. Ils sont souvent complémentaires des autres modes de reconnaissances. Ils peuvent être valorisés par des investigations complémentaires en forages comme l'endoscopie (après nettoyage des parois), l'essai au pressiomètre ou au dilatomètre, les diagraphies différées, la tomographie entre forages. Ils sont également utilisés pour l'installation d'appareillage de suivi piézométrique ou inclinomètres.

\section{- Les fenêtres en parement}

Il s'agit de retirer quelques moellons ou briques du parement et d'observer la maçonnerie, le contact revêtement-terrain, ainsi que le terrain encaissant proche. Pour la réalisation d'une fenêtre en voûte, la procédure impose des précautions pour ne pas trop affaiblir la structure, la dimension maximale est de l'ordre de $0,60 \mathrm{~m} \times 0,60 \mathrm{~m}$ et plusieurs fenêtres ne peuvent être réalisées sur la même section transversale. Elle nécessite la mise en œuvre d'un soutènement préalable pour renforcer la structure, composé de cintres, blindage, ancrages, ou béton projeté (Fig. 3). Dans tous les cas, les fenêtres en voûte doivent être maintenues ouvertes le moins longtemps possible.

Ce type d'investigation, peu prescrit aujourd'hui, a été largement utilisé dans le passé. Leur réalisation est assez facile en piédroit, où elle permet la reconnaissance de l'état des maçonneries et la détermination de la forme des moellons (Fig. 4). Cette technique est actuellement réservée à des reconnaissances ponctuelles en piédroit et dans des structures d'épaisseur inférieure à $0,60 \mathrm{~m}$. Certains grands vides d'extrados ont pu être observés par création de fenêtre.

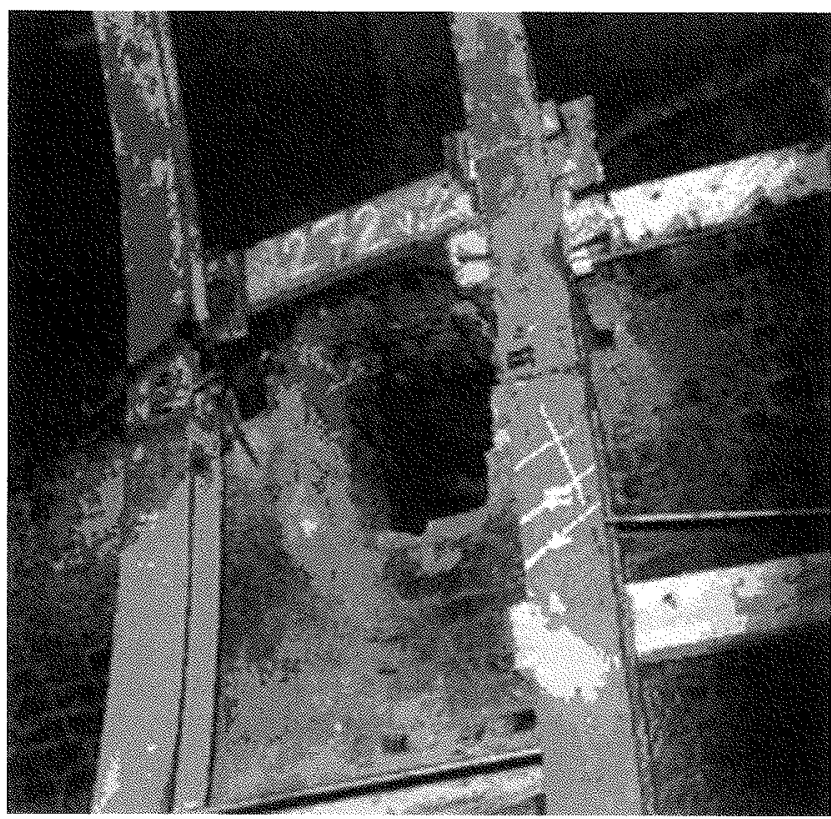

1. Ho. 3 Fenêtre avec protection par cintres. Window with protection by arch.

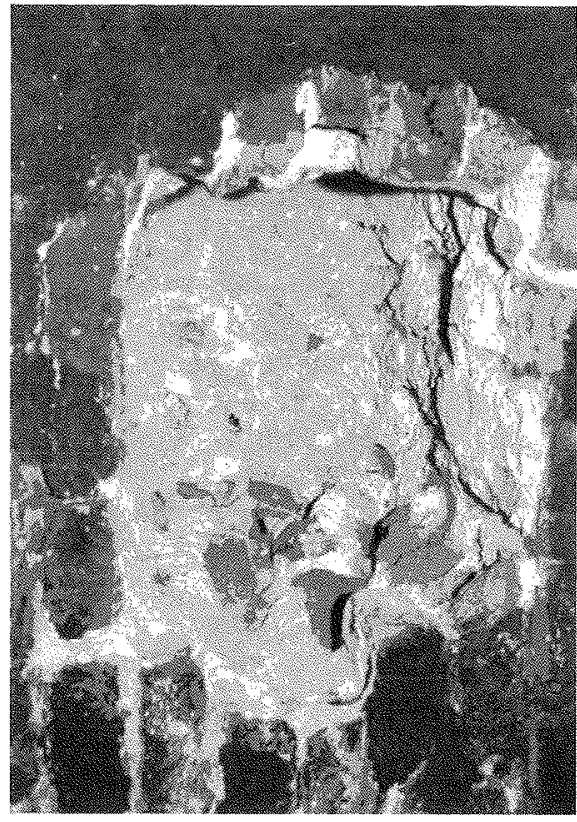

816.4 Fenêtre en piédroit.

Window at abutment.

\section{- Les fouilles en plate-forme}

Les fouilles permettent la reconnaissance du ballast, de la sous couche, de la couche de forme et du terrain d'assise ou du radier, ainsi que l'examen des systèmes d'évacuation des eaux (voir Fig. 5). La plateforme rocheuse ou la surface du radier font partie des éléments du tunnel les plus difficilement observables. Le simple recueil d'éléments géométriques conduit à des opérations lourdes et coûteuses (fouille décapage) où les sujétions imposent une programmation très en amont et une organisation sans faille pour limiter les conséquences sur l'exploitation. Cette observation est cependant possible à moindre coût pendant le renouvellement du ballast. Aussi l'inspection de l'état de la plate-forme et des drains, avec prises de photographies, devrait être systématisée lors de ces opérations.

\section{- Les essais in situ}

Les mesures de contraintes au vérin plat, ou par sur-carottage, qui permettent d'apprécier la contrainte normale en parement du tunnel et de proposer une

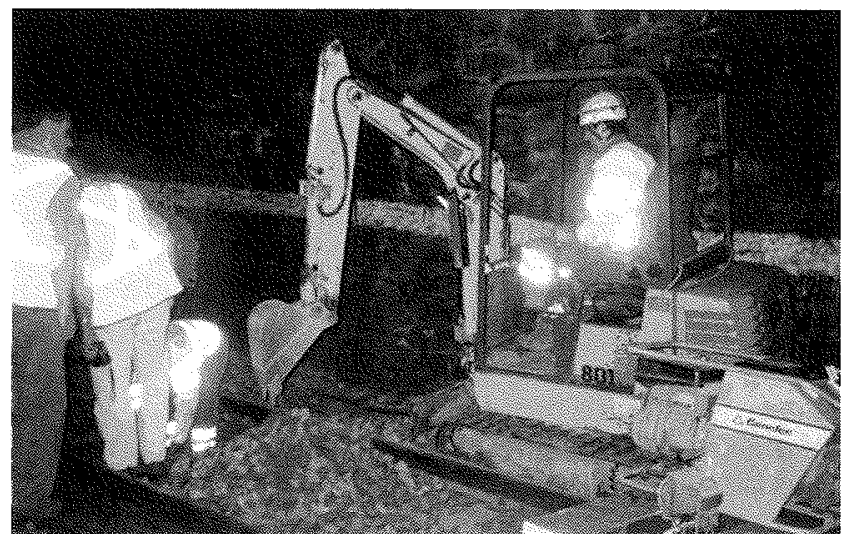

2.6. 5 Fouille mécanisée en plate-forme. Investigation using engine on platform. 
estimation du module d'élasticité en place (Fig. 6), ont cependant une durée relativement longue qui entraine des sujétions importantes d'exécution. Ces essais sont plutôt réservés aux tunnels à gabarit double voie avec une seule voie posée et décalée ou bien sur une coupure prolongée des circulations.

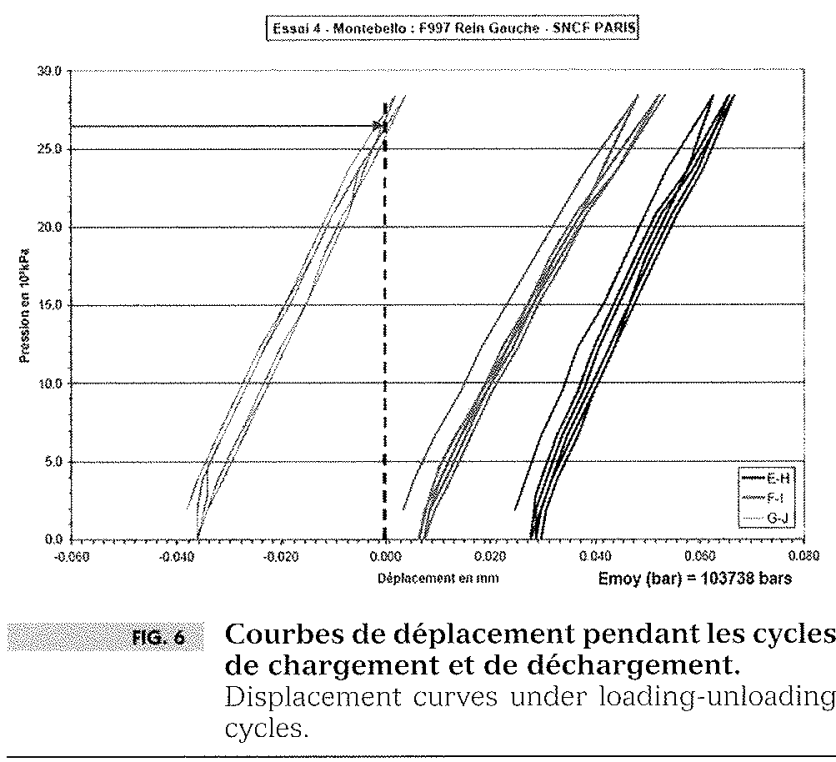

\section{(2)}

\section{Investigations non destructives}

Le radar géologique permet, associé à des sondages carottés pour étalonnage, de repérer la qualité du contact entre revêtement et terrain encaissant, critère important pour la conduite de rescindement. Le radar permet également, en cas d'abaissement de plate-forme, de relever les épaisseurs des structures et d'identifier, toujours en liaison avec des sondages carottés, d'éventuelles discontinuités d'ordre géologi que.

En définitive, c'est la combinaison, au sein d'un programme général de reconnaissances, d'investigations destructives indispensables mais génératrices de fortes gênes pour les circulations de trains, et d'investigations non destructives qui sont sources de résultats moins précis mais ne perturbant pas les circulations, qui permettra de décrire les conditions géotechniques.

La définition de ce programme est une étape cruciale de l'étude géotechnique. Le programme doit en effet être précis et complet, les contraintes d'ex ploitation ne permettant bien souvent aucun complément. L'application de ce programme doit donc être très rigoureuse pour bénéficier de toutes les données demandées. On soulignera notamment le cas de la zone des terrains situés au contact de la structure, qui intervient bien entendu dans la modélisation et les calculs de dimensionnement et dont l'appréciation des caractéristiques se révèle toujours très délicate, compte tenu de l'évolution longue et lente dont ils peuvent être l'objet.

\section{Méthodes de modélisation et lois de comportement}

\section{(5) \\ Considérations générales}

Un nombre important des tunnels, le plus souvent construits entre 1830 et 1910, dont la géométrie doit être adaptée au dégagement d'un nouveau gabarit, peuvent avoir été excavés dans des conditions de terrains peu favorables, avec notamment présence de hors-profils, propension au gonflement, ou état initial de surconsolidation, de sorte que les structures d'origine du tunnel peuvent avoir subi des déformations significatives. Dans tous ces cas de figure, il est important de pouvoir déterminer les conditions de fonctionnement des anciens tunnels, en termes d'interaction sol-revêtement, de propriétés des matériaux constitutifs du terrain et du revêtement (y compris les conditions à court et long termes et les lois de l'évolution connexes), c'est-à-dire d'accéder à l'état de contraintes et aux déformations théoriques du revêtement et des terrains encaissants.

Pour étudier les problèmes d'interaction sol-structures il conviendra de lister les méthodes de calcul, en privilégiant de plus en plus le recours aux méthodes de modélisations numéricues de préférence aux méthodes analytiques, pour prendre en compte les lois non linéaires en contraintes/déformations et la simulation du phasage lors de la construction.

Les phénomènes susceptibles d'influer sur l'interaction sol-revêtement doivent ensuite être recensés afin de pouvoir appréhender (définir et mesurer) les paramètres les plus importants du phénomène concerné. Dans le même temps, les résultats tirés des essais d'identification et de comportement, en laboratoire et in situ sont utilisés. Sont à exploiter les lois comportementales et les modèles types proposés dans la littérature technique pour l'analyse des différents problèmes. En particulier les principaux phénomènes de dégradation et d'endommagement susceptibles d'affecter le comportement à long terme du terrain interagissant avec les structures de revêtement doivent être décrits : - comportement gonflant ;

- comportement contractant;

- phénomènes liés au gradient hydraulique (dont entraînement des fines et modifications des caractéristiques mécaniques des sols).

Les points suivants seront également pris en considération :

- les actions cycliques et les vibrations du sol ;

- le vieillissement des structures de soutènement.

L'ensemble des considérations développées ci-des sus doivent aussi préparer la conception des travaux de mise au gabarit des anciens tunnels, fondée sur les méthodes numériques, conception basée elle aussi sur les différentes méthodes d'analyse, notamment la méthode des différences finies (FDM), la méthode des éléments finis (FEM), et la méthode des éléments distincts (DEM). A cet égard, il faut souligner les avantages potentiels de l'analyse numérique sur les approches plus conventionnelles parfois employées jusqu'alors. 


\section{Cas d'une étude particulière de dégagement de gabarit}

La reconstruction d'un pont à proximité d'un tunnel a conduit à modifier le tracé des voies jusque dans le tunnel et à modifier la section transversale pour dégager la nouvelle implantation des gabarits associés au nouveau tracé de voies.

\section{- Conditions géologiques}

L'ouvrage traverse une série de formations détritiques constituée de cailloux roulés siliceux, d'argiles et sables à graviers, de sable souvent ferrugineux et d'argiles grises à bleuâtres renfermant par place des couches ligniteuses. Ces formations apparaissent assez peu perméables sous les niveaux argileux ; néanmoins, les dépôts sableux et graveleux pourraient renfermer des nappes aquifères.

\section{- Géométrie}

L'étude a été effectuée avec le progiciel de calcul aux éléments finis CESAR-LCPC. La modélisation a été réalisée en deux dimensions, l'ouvrage est alors considéré comme infini dans le sens longitudinal. Cette simplification permet d'éviter d'avoir un maillage trop important et donc des temps de calculs trop grands. De plus, cette hypothèse nous place du côté de la sécurité puisqu'elle ne prend pas en compte le phasage par plots des travaux.

Le revêtement du tunnel, le ballast, les structures mises en place lors des travaux, ainsi que le massif environnant, ont été modélisés à l'aide d'éléments surfaciques. Chaque partie qui interviendra à différentes étapes du phasage (voûte parapluie, partie rescindée, coque en BP, etc.) est représentée par un groupe indépendant. Les extensions des modèles sont choisies afin de respecter les prescriptions courantes dans une modélisation aux éléments finis. Le maillage, zoomé au niveau de l'ouvrage, est présenté sur la figure 7.
- Lois de comportement et caractéristiques mécaniques

Les paramètres mécaniques du terrain encaissant et de la maçonnerie sont tirés en grande partie du rapport d'étude géotechnique. Les caractéristiques mécaniques de la coque BP et du ballast sont issues soit de la littérature, soit d'autres études numériques dans lesquelles ces matériaux ont été utilisés.

Pour la demi-voûte parapluie et la structure nouvelle, un calcul de caractéristiques écuivalentes a été réalisé. En effet, il s'agit d'assemblage de plusieurs matériaux (tubes pétroliers et coulis pour la demi-voûte parapluie, cintres réticulés et béton pour la structure nouvelle) qui ont été modélisés comme un matériau unique équivalent avec des propriétés calculées à partir des caractéristiques de chaque composant rapportées à leur inertie. Pour la zone de sol renforcé, nommé ( sable argileux injecté », des calculs ont été réalisés avec une fourchette haute $(\mathrm{FH})$ et une fourchette basse (FB) pour le module d'Young $E$, correspondant à différents stades d'efficacité de la consolidation du terrain par injection de coulis. Les lois de comportement et les paramètres qui ont été utilisés lors des modélisations sont présentés dans le tableau I.

\section{- Phasage choisi pour les calculs}

Les phases, ainsi que les taux de déconfinement $\lambda$ choisis à chaque phase, ont été déterminés en réalisant plusieurs calculs et en faisant varier certains paramètres afin de trouver la combinaison la plus réaliste tout en restant du côté de la sécurité.

Les phases de calcul sont les suivantes (Fig. 8) :

- phase 1 : création de l'état de contraintes initial en appliquant les contraintes géostatiques avec $\mathrm{K}_{0}=0,5$ et creusement du tunnel en appliquant le principe de la méthode convergence - confinement avec un taux de déconfinement de $\lambda=0,4$;

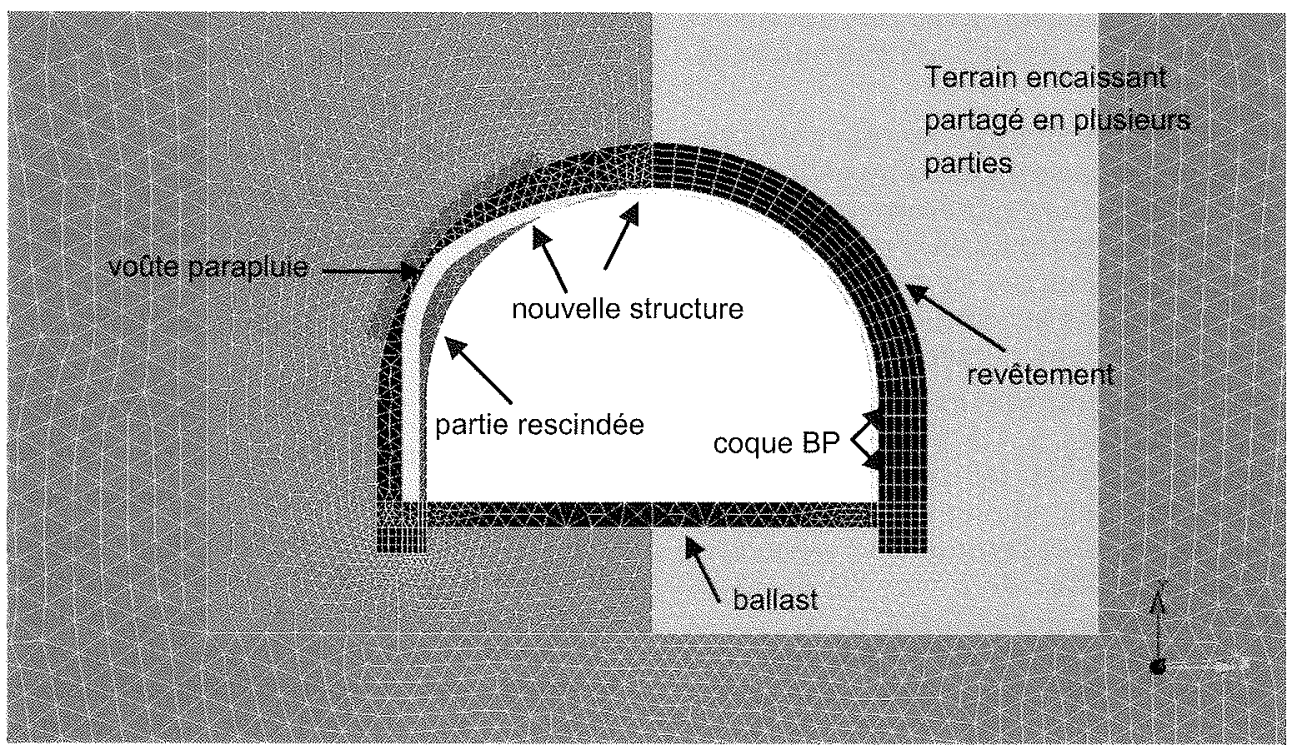


TABufuI Lois de comportement et paramètres choisis pour les matériaux. Constitutive model and parameters chosen for the materials.

\begin{tabular}{|c|c|c|c|c|c|c|c|c|c|}
\hline (y) & 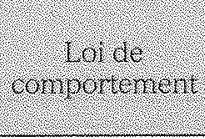 & 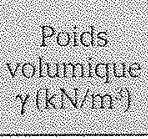 & 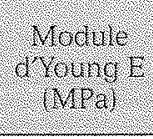 & 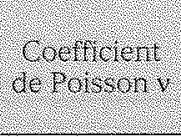 & 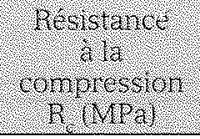 & 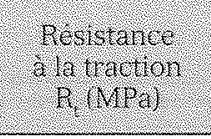 & 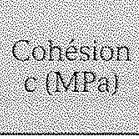 & 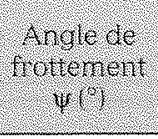 & 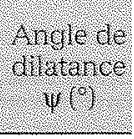 \\
\hline $\begin{array}{c}\text { Sable argileux } \\
\text { zone autour } \\
\text { tunnel }\end{array}$ & $\begin{array}{l}\text { Mohr } \\
\text { Coulomb }\end{array}$ & 19,0 & 1,4 & 0,33 & & & 0,004 & 32 & 2 \\
\hline $\begin{array}{l}\text { Sable argileux } \\
\text { loin du tunnel }\end{array}$ & $\begin{array}{l}\text { Mohr } \\
\text { Coulomb }\end{array}$ & 19,0 & 14 & 0,33 & & & 0,004 & 32 & 2 \\
\hline $\begin{array}{c}\text { Sable argileux } \\
\text { injecté }\end{array}$ & $\begin{array}{l}\text { Mohr } \\
\text { Coulomb }\end{array}$ & 19,0 & $\begin{array}{l}\mathrm{FB}: 5 \\
\mathrm{FH}: 14\end{array}$ & 0,33 & & & 0,004 & 32 & 2 \\
\hline $\begin{array}{c}\text { Maçonnerie } \\
\text { de moellons } \\
\text { calcaires }\end{array}$ & Élasticité & 26,0 & 10000 & 0,2 & $3,2<R_{c}<42,6$ & $\begin{array}{c}\left(1<\mathrm{R}_{\mathrm{t}}<1,5\right) \\
\text { valeur estimée }\end{array}$ & & & \\
\hline Ballast & $\begin{array}{l}\text { Mohr } \\
\text { Coulomb }\end{array}$ & 20,0 & 3,5 & 0,2 & & & 0,2 & 50 & 0 \\
\hline Voûte parapluie & $\begin{array}{l}\text { Mohr } \\
\text { Coulomb }\end{array}$ & 19,0 & 4000 & 0,33 & & & 0,774 & 32 & 2 \\
\hline $\begin{array}{c}\text { Nouvelle } \\
\text { structure } \\
\text { avec cintres }\end{array}$ & Élasticité & 25,0 & 200000 & 0,2 & & & & & \\
\hline Coque BP & Élasticité & 23,0 & 10000 & 0,2 & & & & & \\
\hline
\end{tabular}

- phase 2 : application du reste du déconfinement $\lambda=0,6$ et mise en place du revêtement et du ballast en appliquant leur poids propre ;

- phase 3 : mise en place ou non (selon le calcul et de façon combinée ou non) de la demi-voûte parapluie et de la zone de sol renforcée par attribution des caractéristiques améliorées aux groupes concernés et rescindement du côté gauche de l'ouvrage en appliquant des forces de cléconfinement sur le pourtour de la partie rescindée avec un taux $\lambda=0,7$ et des contraintes ayant comme origine la phase 2 ;

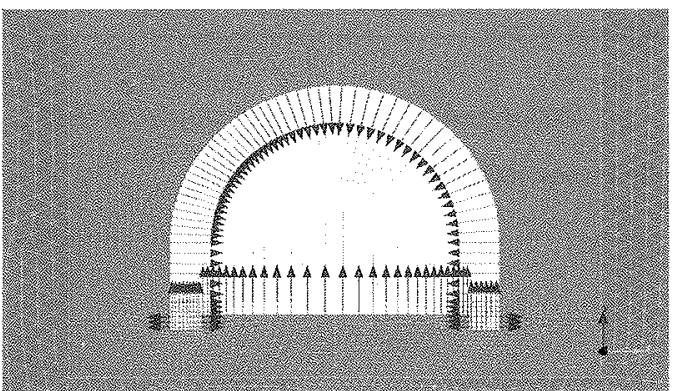

Phase 1 (première étape de la construction)

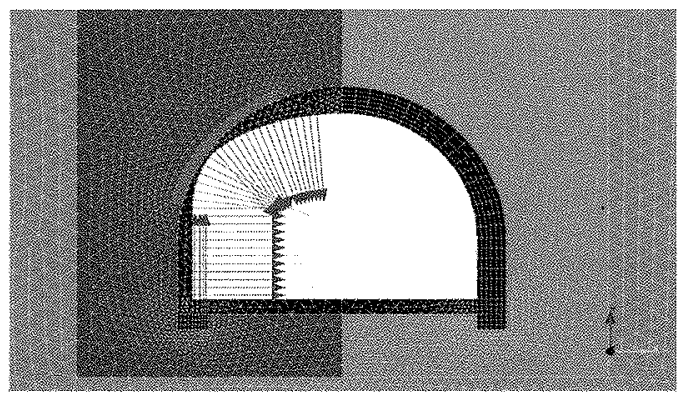

Phase 3 (première étape des travaux)
- phase 4 : mise en place de la nouvelle structure composée de cintres réticulés et de béton et de la coque en BP (attribution des caractéristiques et application du poids propre) et application du reste des forces de déconfinement dues au rescindement avec un taux $\lambda=0,3$.

Les résultats sont présentés et analysés dans les paragraphes suivants en fonction de la phase de calcul et des hypothèses choisies.

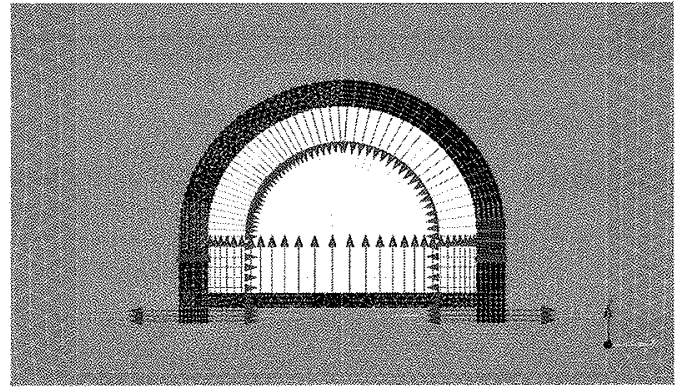

Phase 2 (fin de la construction)

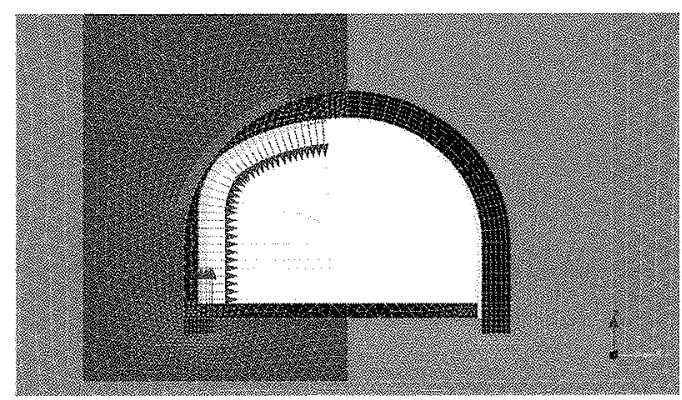

Phase 4 (dernière étape de la construction)

1. Ho 8 Schéma des quatre étapes de calcul. Schems of the 4 stages of calculation. 
Fin de la construction (phase 2)

Comme nous ne disposons pas d'éléments permettant de caler le modèle numérique avec la réalité, un état de contraintes dans le revêtement cohérent (en fonction de la hauteur de couverture, du poids volumique et du module d'Young $E$ du terrain) a été recherché. Les déplacements obtenus sont assez importants ( $8,5 \mathrm{~cm}$ en clé par exemple) mais ils sont cumulés sur les deux phases de construction et ne s'appliquent pas en totalité sur la maçonnerie. Ils restent donc, vu la faible hauteur de couverture et la mauvaise qualité du terrain encaissant. Les contraintes de compression sont de l'ordre de -2,4 MPa en intrados de la voûte et de - 0,5 MPa en piédroits (intrados et extrados). Une certaine traction s'est développée à l'extrados de la maçonnerie au niveau de la voûte $1,32 \mathrm{MPa}$ en clé et 1,48 MPa en rein droit). Ces valeurs sont acceptables et correspondent aux déplacements obtenus. La figure 9 montre les contraintes de compression et les vecteurs de déplacement.

\section{Première phase des travaux de rescindement (phase 3)}

Cette phase est la plus critique pour le revêtement du tunnel car l'épaisseur de la maçonnerie est fortement diminuée et la nouvelle structure n'est pas encore mise en place. Un taux de déconfinement assez important $(\lambda=0,7)$ a été choisi pour se placer dans une situation relativement défavorable. Un premier cas a été réalisé sans travaux préalables au rescindement. Ensuite plusieurs solutions de renforcement ont été testées et combinées. Les cas les plus importants sont présentés ci-après.

- 1 er cas : sans renfort de sol et sans demi-voûte parapluie

Des tractions importantes se développent dans la maçonnerie restante : 3,3 $\mathrm{MPa}$ en intrados et 4,0 $\mathrm{MPa}$ en extrados. Des déplacements de $2,7 \mathrm{~cm}$ se produisent. Au vu de ces résultats, il est possible d'affirmer que cette phase intermédiaire des travaux n'est pas stable. En effet, la limite en traction d'une maçonnerie de moellons est souvent comprise entre 1 et 1,5 MPa. Et des céplacements de l'ordre de plusieurs centimètres ne sont pas acceptables et peuvent entraîner des désordres importants. La figure 10 montre les contraintes de traction et les vecteurs de déplacement

Des travaux de renforcement préalables aux travaux de rescindement ont été envisagés pour remédier aux problèmes rencontrés dans le premier cas. En fonction des conditions de réalisation, d'accès, de mise en œuvre, deux techniques sont proposées : le renforcement du sol par injection autour du tunnel du côté gauche où aura lieu le rescindement et la création d'une demi-voûte parapluie qui viendra renforcer la maçonnerie localement, là où elle sera le plus affaiblie. Dans les calculs suivants, les deux solutions techniques

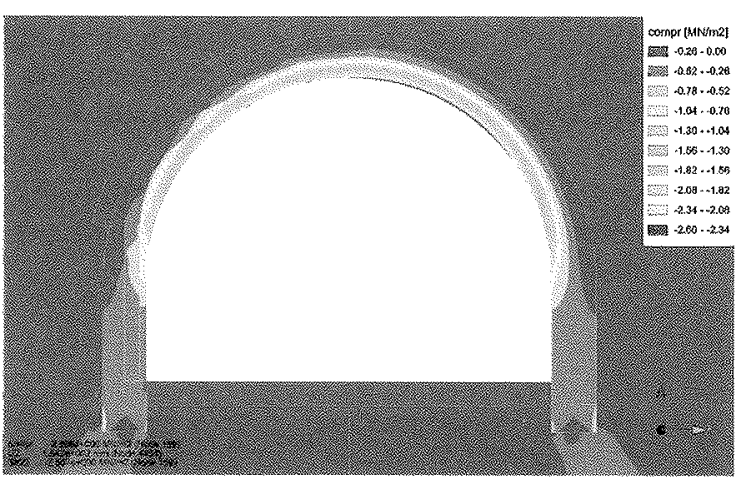

Contraintes de compression

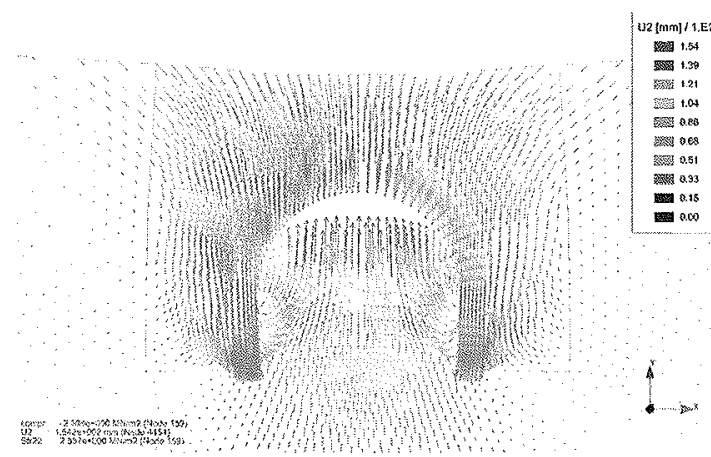

Déformée et vecteurs de déplacement

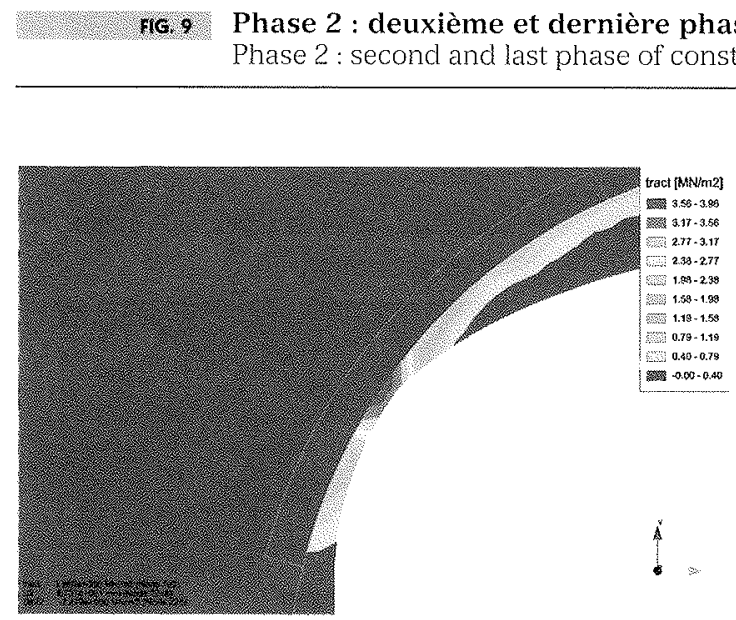

Contraintes de traction

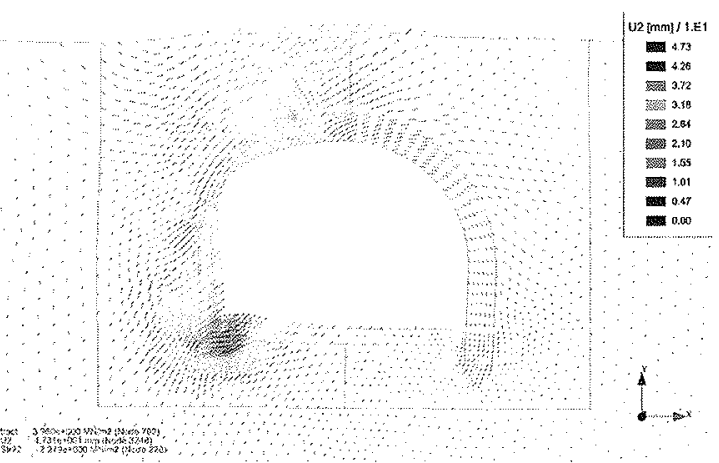

Déformée et vecteurs de déplacement

FG. 10 Phase $3,1^{\text {er }}$ cas : première phase des travaux de rescindement sans renfort de sol et sans demi-voûte parapluie.

Phase 3, first case : first phase of splitting work without reinforcement of soil and without half vault umbrella. 
ont été testées de façon séparée et combinée dans le but d'étudier leur influence et de choisir le renforcement le plus adapté. Le module d'Young du sol renforcé n'étant pas connu de façon très précise, il a été pris égal à trois valeurs : une valeur faible de $5 \mathrm{MPa}$, une valeur moyenne de $10 \mathrm{MPa}$ et une valeur plutôt forte de $14 \mathrm{MPa}$.

- $2^{e}$ cas : avec sol renforcé $E=14 \mathrm{MPa}$ et sans demivoûte parapluie

Dans ce cas, les tractions qui se développent dans la maçonnerie restante sont diminuées par rapport au cas précédent mais elles restent tout de même trop importantes : 1,7 $\mathrm{MPa}$ en intrados et 2,0 $\mathrm{MPa}$ en extrados. Des déplacements de l'ordre de $1,0 \mathrm{~cm}$ sont obtenus. Ils ont été bien réduits par la mise en œuvre du sol renforcé et sont alors acceptables. La figure 11 montre les contraintes de traction et les vecteurs de déplacement dans le $2^{\mathrm{e}}$ cas.

- $3^{e}$ cas : sans renfort de sol et avec demi-voûte parapluie

Ici, les tractions dans le revêtement rescindé ont aussi été réduites : 1,7 MPa en intrados et 1,6 MPa en extrados mais elles restent tout de même trop élevées. Les déplacements sont de l'ordre de $2,5 \mathrm{~cm}$ et sont donc trop importants.
- $4^{e}$ cas : avec sol renforcé $E=14 \mathrm{MPa}$ et avec demivoûte parapluie

Dans ce cas, les contraintes de traction dans la maçonnerie restent admissibles : 0,6 $\mathrm{MPa}$ en intrados et $0,5 \mathrm{MPa}$ en extrados. Les déplacements sont légèrement inférieurs à $1,0 \mathrm{~cm}$. La figure 12 montre les contraintes de traction et les vecteurs de déplacement dans le $4^{\mathrm{e}}$ cas.

- $5^{e}$ et $6^{e}$ cas : avec sol renforcé $E=10$ ou $5 \mathrm{MPa}$ et avec demi-voute parapluie

Dans ces deux cas, les contraintes de traction dans la maçonnerie augmentent un petit peu par rapport au cas précédent dans lequel le module du sol renforcé était de $14 \mathrm{MPa}$. Les valeurs restent, tout de même, dans des limites acceptables. Pour un sol renforcé avec un module d'Young de $5 \mathrm{MPa}$, la contrainte de traction en intrados de la partie rescindée est de 0,8 $\mathrm{MPa}$

De la même façon, les déplacements sont un peu plus importants avec une valeur de $1,2 \mathrm{~cm}$ au niveau du rein gauche pour le cas où le sol renforcé possède un module de $5 \mathrm{MPa}$.

\section{Deuxième phase des travaux de rescindement (phase 4)}

Les valeurs obtenues ne sont pas détaillées pour chaque cas dans ce paragraphe car elles suivent les

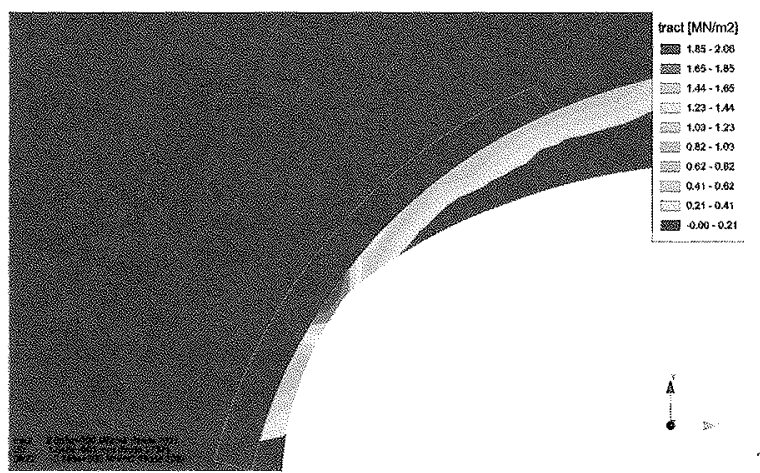

Contraintes de traction

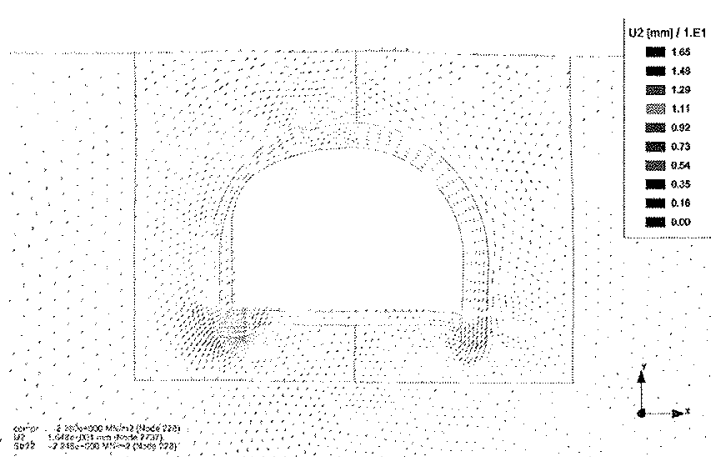

Déformée et vecteurs de déplacement

F6. 11 Phase $3,2^{\mathrm{e}}$ cas : première phase des travaux de rescindement avec renfort de sol $\mathrm{E}=14 \mathrm{MPa}$ et sans demi-voûte parapluie.

Phase 3, second case : first phase of splitting work with reinforcement of soil $\mathrm{E}=14 \mathrm{MPa}$ and without half vault umbrella.

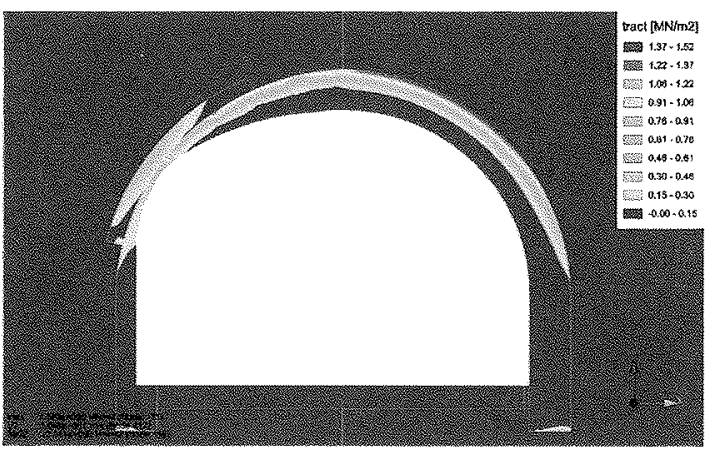

Contraintes de traction

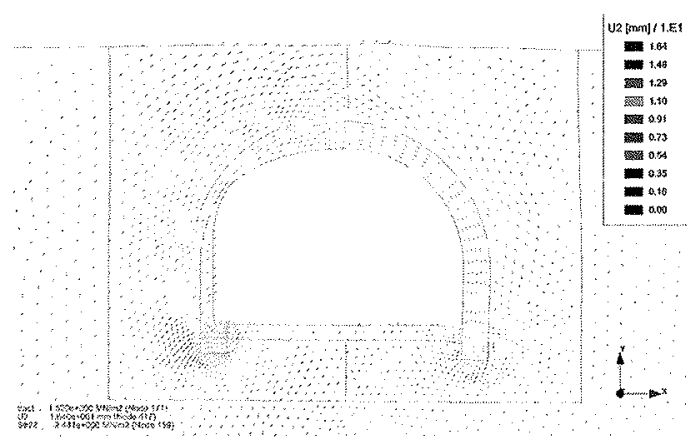

Déformée et vecteurs de déplacement

tia. 12 Phase $3,4^{\mathrm{e}}$ cas : première phase des travaux de rescindement avec renfort de sol $E=14 \mathrm{MPa}$ et avec demi-voûte parapluie.

Phase 3 , fourth case : first phase of splitting work with reinforcement of soil $E=14 \mathrm{MPa}$ and with half vault umbrella. 
tendances présentées dans le paragraphe précédent pour la phase 3 . Par exemple, la contrainte de traction dans la maçonnerie en intrados du rein gauche passe de $3,4 \mathrm{MPa}$ dans le $1^{\mathrm{er}}$ cas (sans renfort de sol et sans demi-voûte parapluie) à $0,6 \mathrm{MPa}$ dans le $4^{c}$ cas (avec renfort de sol $E=14 \mathrm{MPa}$ et avec demi-voûte parapluie) et à $0,8 \mathrm{MPa}$ dans le $6^{\mathrm{e}}$ cas (avec renfort de sol $\mathrm{E}=5 \mathrm{MPa}$ et avec demi-voûte parapluie).

Qu'il y ait renforcement ou non et quel que soit le type de renforcement (sol injecté et/ou demi-voûte parapluie), les valeurs des contraintes dans la nouvelle structure sont tout à fait acceptables. Les valeurs de la contrainte de compression en intrados au niveau du rein gauche varient de $-1,8 \mathrm{MPa}$ à - 1,4 $\mathrm{MPa}$ selon le cas. Des tractions se développent à l'extrados de la structure formée de cintres et de béton (reprise d'une partie de celles venant de la maçonnerie) tout en restant admissibles : entre 1,0 et 1,5 $\mathrm{MPa}$.

D'autres calculs ont été réalisés pour étudier l'influence de la raideur de la nouvelle structure mise en place. La valeur du module d'Young, calculée en fonction du nombre de cintres mis en place, a été prise entre $50000 \mathrm{MPa}$ et $200000 \mathrm{MPa}$. Les résultats ont montré que la valeur la plus forte était nécessaire (c'est-à-dire quatre cintres par mètre) pour garder des déplacements et des contraintes en phase finale acceptables pour le revêtement existant de l'ouvrage.

Les figures 13,14 et 15 montrent les trois cas avec les résultats les plus caractéristiques pour cette phase.

Même si on rappelle que les modélisations sont assez défavorables (modèle 2D ne prenant pas en compte le travail par plots alternés et taux de déconfinement en phase 3 assez élevé), les valeurs de traction obtenues dans la maçonnerie en phase provisoire de travaux sont trop importantes $(3,9 \mathrm{MPa})$ si aucune précaution particulière n'est prise. Les déplacements de l'ordre de $2,7 \mathrm{~cm}$ sont également rédhibitoires.

Le cas réalisé avec le renfort de sol à gauche de l'ouvrage uniquement (cas $n^{\circ} 2$ ) indique que ce traitement n'est pas suffisant à lui seul. En effet, bien que les déplacements soient réduits de façon notable, il reste des tractions dans le revêtement existant de l'ordre de $2 \mathrm{MPa}$, ce qui dépasse la limite acceptable.

Dans le calcul mené avec la voûte parapluie uniquement (cas $n^{\circ} 3$ ), les tractions sont réduites (passage de $4 \mathrm{MPa}$ à 1,6 MPa en extrados de la maçonnerie en rein gauche en phase 3) mais de façon insuffisante.

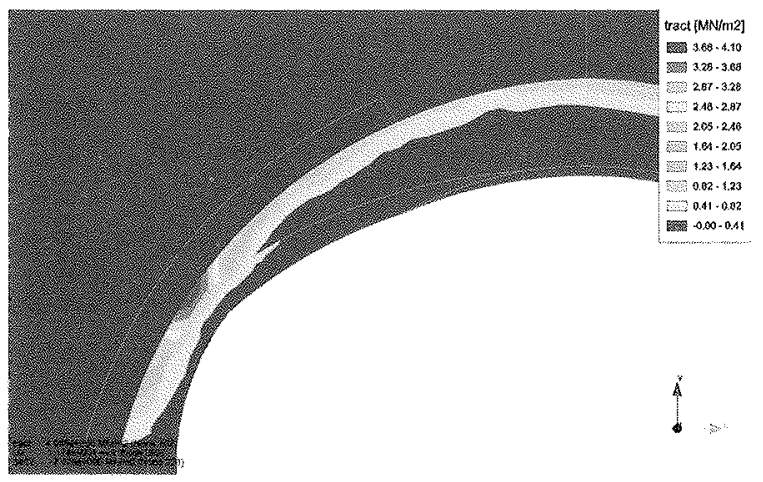

Contraintes de traction

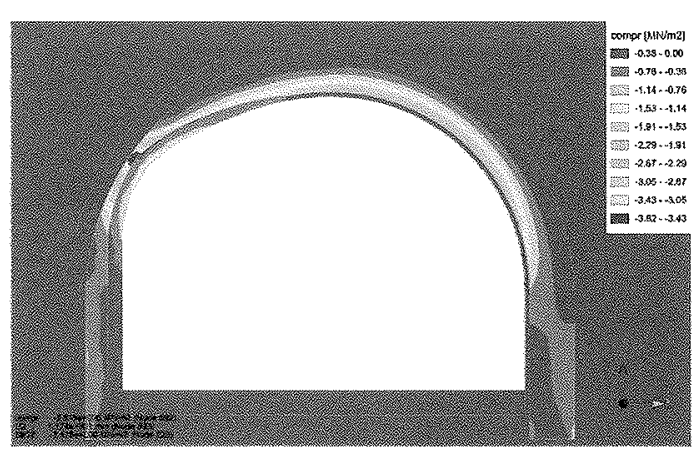

Contraintes de compression

70. 13. Phase 4, $1^{\text {er }}$ cas : deuxième phase des travaux de rescindement sans renfort de sol et sans demi-voûte parapluie.

Phase 4, first case : second phase of splitting work without reinforcement of soil and without half vault umbrella.

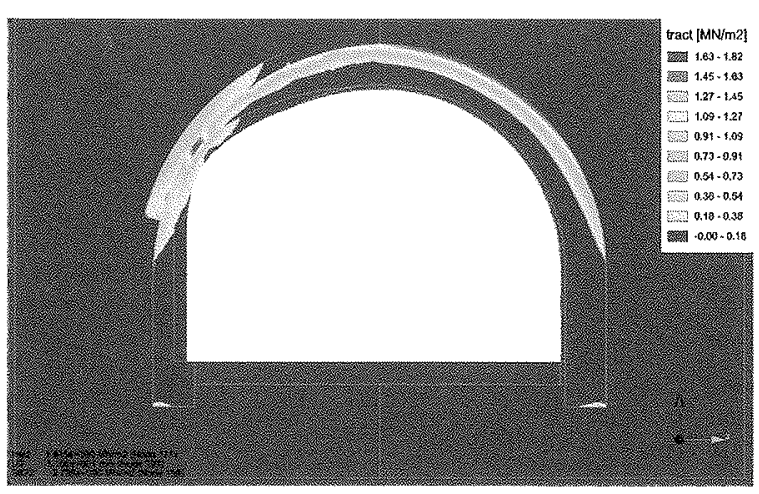

Contraintes de traction

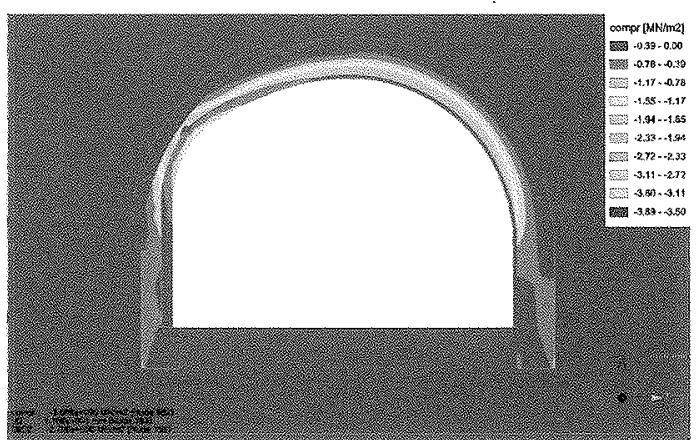

Contraintes de compression

2. If 14 Phase $4,3^{\mathrm{e}}$ cas : deuxième phase des travaux de rescindement sans renfort de sol et avec demi-voûte parapluie.

Phase 4 third case : second phase of splitting work without reinforcement of soil and with half vault umbrella. 


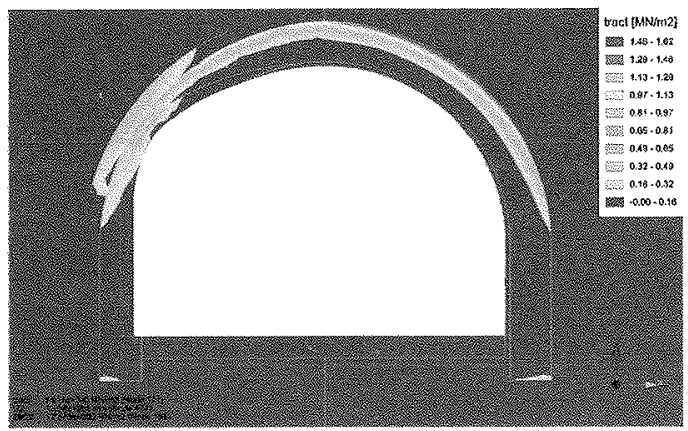

Contraintes de traction

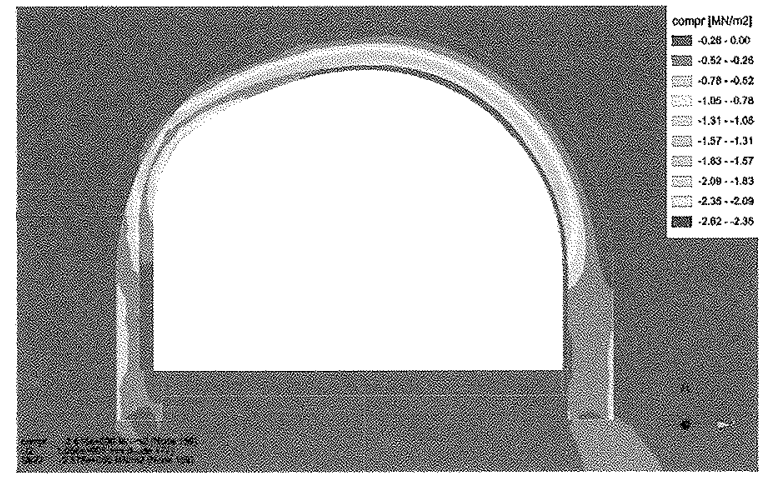

Déformée et vecteurs de déplacement

FG. 15 Phase $4,6^{\mathrm{e}}$ cas : $2^{\mathrm{e}}$ phase des travaux de rescindement avec renfort de sol E $=5 \mathrm{MPa}$ et avec demi-voûte parapluie.

Phase 4, sixth case : second phase of splitting work with reinforcement of soil $E=5 \mathrm{MPa}$ and with half vault umbrella.

Les déplacements quant à eux sont très peu influencés (passage de $2,7 \mathrm{~cm}$ à 2,5 cm) et restent donc trop importants.

Les résultats des différents calculs menés montrent qu'il est nécessaire de réaliser de façon combinée les deux solutions de renforcement envisagées (consolidation du terrain encaissant par injection et réalisation d'une demi-voûte parapluie) avant d'effectuer le rescindement en rein gauche du tunnel de Mousserolles. La combinaison des deux méthodes de renforcement montre des résultats satisfaisants. Quelle que soit la valeur du module d'Young du sol renforcé, variant entre $5 \mathrm{MPa}$ et $14 \mathrm{MPa}$, les contraintes de traction dans la maçonnerie restant en phase 3 sont acceptables. Les déplacements allant de 0,9 à $1,2 \mathrm{~cm}$ sont également admissibles.

\section{5}

\section{Conclusion}

Les contraintes ferroviaires pesant sur un projet de mise au gabarit de tunnel ferroviaire sont très lourdes: elles obligent à adapter l'organisation de ces travaux aux obligations de perturbation minimale, voire de maintien quasi intégral du trafic existant. Cette adaptation passe par la recherche de découpage des travaux en phasage complexe, dont le respect strict permet le maintien en sécurité des circulations.

Les études géotechniques doivent être capables non seulement d'utiliser les données les plus représentatives de l'environnant, malgré les incertitudes portant sur le véritable comportement des terrains au contact des structures d'origine, mais également de modéliser ces différentes phases de façon à connaître les sollicitations lors des phases provisoires comme lors de la phase d'achèvement des travaux. 